\title{
Monitoring motor nerve function in leprosy patients
}

\author{
J. WIM BRANDSMA \\ Schieffelin Leprosy Research \& Training Centre, Karigiri, 632 106, \\ Tamil Nadu, India
}

\author{
Accepted for publication 1 August 2000
}

\begin{abstract}
Summary Manual muscle strength testing has an important function in the management of leprosy patients. Its importance was first recognized in the 1960s, especially when following patients who were started on steroid treatment to monitor the nerve function and the effect of treatment. In those days, and still in many centres today, many or all muscles were tested that are innervated by the nerves that can be at risk in leprosy. The author argues that not all muscles innervated by the nerves at risk need to be tested and also that many muscles cannot be tested in isolation. A muscle charting form is presented which is suitable for screening purposes, and that also allows for more detail when motor function is impaired.
\end{abstract}

\section{Introduction}

Assessment and evaluation of motor function is very important in the detection of early motor nerve function impairment. When nerve function impairment is detected early it is often reversible. Changes in nerve function impairment are of ten taken into account when deciding on doses and duration of corticosteroid treatment. In addition, the (changing) status of motor nerve function is taken into consideration when deciding on tendon transfer surgery. For these reasons it is important to have a muscle charting form that lists muscles/movements that can be tested and graded and that will give reliable information for decision making. It should be realized that manual muscle strength testing, and also sensory testing, is a 'proxy' for nerve function evaluation which can only be done with electroneurophysiological equipment. In other words, by grading muscle strength we indirectly obtain information about the primary function of the nerve, which is conduction of electrical impulses.

Goodwin was the first to write about the importance of muscle charting in leprosy neuritis. ${ }^{1}$ The charting was elaborate. All muscles supplied by the nerves that could be affected in leprosy were listed and the naming and listing of the individual muscles implied that they could all be graded. It has been shown that in most movements often more than one muscle contributes to the movement and the strength when testing for resistance. ${ }^{2,3}$ In a later

Correspondence to: J. W. Brandsma, International Nepal Fellowship, Green Pastures Hospital, PO Box 5, Pokhara, Nepal (e-mail: wbrandsm@inf.org.np) 
paper, only those movements were listed that could be graded and would allow for easy evaluating and monitoring of motor function changes in leprosy patients. ${ }^{4}$ The purpose of this paper is to discuss the tests that should and could be done. For details about the testing itself, the reader is referred to the aforementioned publication. A form is presented that is suitable for screening purposes or for 'follow-up' with more detail for subjects with established motor function impairment to evaluate and monitor changes over time. This paper will not discuss muscle tests that may be relevant from a (reconstructive) surgical point of view. Specific surgery forms should be used for subjects that are considered for reconstructive surgery.

\section{Detail of grading}

The Medical Research Council 6-point scale (0-5) is nowadays the most commonly used to assess and evaluate muscle strength. ${ }^{5}$ The grades are defined by range of motion, degree of resistance that can be overcome and whether or not the limb (segment) moves against gravity (Table 1). The grading is very useful in the evaluation of patients who are treated for recent nerve function impairment to monitor the effect of instituted treatment(s). The grading of muscle strength needs a certain level of understanding of anatomy, standardization of testing procedures and experience. Some authors felt that this was not always present in 'field' leprosy control work and they proposed tests that could be easily performed by the fieldworker or copied by the subjects. ${ }^{6-8}$ The distinct disadvantage of these tests is that minor degrees of weakness will not be detected. Patients with 'minor' degrees of weakness will still be able to perform these tests. In other words, most of these tests will first become 'positive' when there is considerable weakness of the muscles present. If early detection of nerve function loss improves prognosis for preservation of nerve function tests should be used that test for resistance. Such tests will more likely reveal early motor function impairment.

\section{Facial nerve}

The facial nerve innervates all muscles of facial expression. Most commonly, the upper branch is affected which will result in weakness or paralysis of the muscle that closes the eye. Grading with the MRC scale will be possible except maybe for grade 1. In my experience, the testers find it difficult to agree on a grade 1 . There is good agreement between the testers on a

Table 1. Medical Research Council (MRC) muscle grading scale

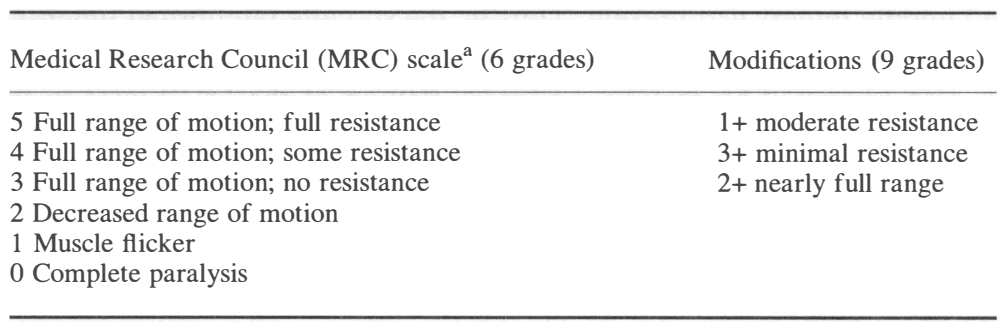

\footnotetext{
${ }^{\text {a }}$ The MRC scale originally grades $4-5$ against gravity and $0-3$ with gravity eliminated. For muscle grading of small muscles the effect of gravity is negligible.
} 
grade 0 (inability to close the eye) and grade 2, partial closure. For this reason it is now being considered not to use grade 1 for eye closure.

In light eye closure the subject is asked to close his eye as if falling asleep. An eyelid gap, if present, is then measured. This measurement is indicated for subjects with strength less then grade 3. It is another way of evaluating changes in strength avoiding the compensatory movements that subjects often use by pulling up their cheeks to achieve full closure.

The 'empty' rows on the muscle charting form (Figure 1) can be used to list and grade one or two additional muscles in case there is a total facial palsy. Weakness or paralysis of the frontalis muscle, responsible for raising the eyebrow, does occasionally occur in leprosy patients. This could also be indicated in the empty row. Using the MRC grades for strength evaluation of this muscle, however, is not practical.

\section{Ulnar nerve}

In a separate paper, the author has discussed in more detail the testing and grading of the intrinsic muscles of the hand. ${ }^{9}$ With six tests the motor function of the ulnar nerve can be evaluated and monitored: abduction of the little and index fingers and intrinsic position of the four fingers.

The main contributing muscle towards abduction of the little finger is possibly the abductor digiti minimi. With respect to its function, it could be considered a dorsal interosseus (flexion and abduction of the (fifth) metacarpophalangeal joint). The hypothenar muscle mass is easily palpable to be able to give a grade 1 or 2 . This is also the case for abduction of the index finger for which the muscle mass (first dorsal interosseus) is easily palpable in the first webspace. When in doubt about the presence of a very weak first dorsal interosseus (grade 1-2), the examiner could ask the patient to strongly pinch against the index finger preferably in a pulp to pulp pinch with the thumb in opposition. The first dorsal interosseus muscle may then become palpable.

The interossei are the muscles responsible for MCP stabilization and they are tested in the so-called 'lumbrical' position/test. This has been discussed more extensively elsewhere by the author but the main evidence will be summarized here. ${ }^{9}$

In an isolated ulnar palsy there is always 'latent', sometimes called 'hidden', clawing of the index and middle finger. Only in the presence of a Martin-Gruber anastomosis or localized damage of the ulnar nerve at the level of the wrist may near normal strength still be present in the intrinsic position (MCP flexion, IP extension) of the index and to a lesser degree of the middle finger. ${ }^{10-14}$ In long standing ulnar palsy 'overt' four finger clawing will become evident. Secondly, the author has never seen cases of low median palsy in which clawing of the index and middle fingers was present. Thirdly, the so-called lumbrical function is restored by 'reactivating' the interossei by direct insertion of tendon grafts into the interossei tendons. 'Clawing' is also corrected by restoring primary flexion of the MCP joint in the so-called Zancolli Lasso operation. No leprosy surgeon, I would hope, would do only a two-finger (ring and small fingers) 'claw' correction in an ulnar palsy. The author has seen cases in which, in the early days of tendon transfer surgery, only the ring and little fingers were corrected. When reviewing these patients years later the index and middle fingers showed clawing!

It can be very misleading in a muscle charting form to have the 'lumbrical' test for the index and middle fingers under the 'heading' median nerve. An isolated palsy of the ulnar nerve will show weakness and, often at a much later stage, paralysis of the 'lumbricals' of the 


\begin{tabular}{|l|l|l|l|l|l|l|l|l|l|l|}
\hline \multicolumn{2}{|c|}{ Green Pastures Hospital } \\
Voluntary Muscle Testing \\
\hline Name \\
\hline
\end{tabular}

First assessment weakness/paralysis in red; following assessments only deterioration in red. Always note duration of (new) muscle weakness/paralysis

\begin{tabular}{|l|l|}
\hline Date: & Comments: \\
\hline & \\
\hline & \\
\hline & \\
\hline & \\
\hline & \\
\hline & \\
\hline
\end{tabular}

MRC scale: $5=$ normal range of motion (ROM) and strength; $4=$ normal ROM reduced strength; $3=$ normal ROM no resistance; $2=$ partial ROM no resistance; $1=$ muscle flicker only; $0=$ paralysed 
index and middle finger. Most therapists and medical doctors alike will attribute this to anomalous innervation or, worse still, to partial median nerve involvement. It seems therefore justified to include this test for the interossei (preferred term 'intrinsic test') for all four fingers listed with the ulnar nerve.

There are no clinically reliable tests by which the strength of the lumbricals can be graded on the MRC scale.

Differences in grades between ulnar innervated muscles on the radial side of the hand versus the ulnar side may be explained on account of a Martin-Gruber anastomosis or localized damage at near or at the ulnar carpal tunnel (Guyon's canal).

Two extrinsic muscles are innervated by the ulnar nerve: the flexor digitorum profundus to the ring and small finger and the flexor carpi ulnaris. Testing of these muscles could be of interest from a prognostic point of view. Do patients with a 'low' ulnar palsy have a better chance for recovery?

The empty rows on the form could be used to record the outcome of any alternative 'tests' (see Discussion). For screening purposes, we advise testing abduction of the little finger. When weakness or paralysis is detected, the other movements are also tested.

\section{Median nerve}

The median nerve is most commonly affected at the level of the wrist, rarely in the cubital fossa (level of the elbow). When the nerve is affected at the wrist there will be weakness or paralysis of the muscles that oppose the thumb: abductor pollicis, opponens pollicis and to a variable degree, depending on its pattern of innervation, the flexor pollicis brevis. In addition, the median nerve innervates the lumbrical muscles to the index and middle fingers. As argued above, the interossei are the muscles tested in the so-called 'lumbrical' test.

The only two tests to evaluate the motor function of the median nerve are abduction and opposition of the thumb. Neither movement can be attributed to the action of one muscle only.

For screening purposes, we advise to test abduction of the thumb. Other tests should also be done if this test reveals weakness. It could then also be of interest to check if there is weakness of the extrinsic muscles that are innervated by the median nerve. For this, we suggest that thumb IP flexion is tested (flexor pollicis longus) and/or the flexor digitorum superficialis of the index finger.

\section{Radial nerve}

The radial nerve is rarely involved in leprous neuropathy. In leprosy, when there is motor function impairment of the radial nerve, there often will be associated motor function impairment of the ulnar and median nerves. This is often referred to as a triple (=3) palsy. However, isolated motor function impairment of the radial nerve does occur. If a patient is making use of crutches a 'crutch palsy' (neuropraxia), because of the wrong use of crutches, should be ruled out!

For screening purposes, it is sufficient to test the combined wrist extensors in extension of the wrist. Only when weakness or paralysis is detected in this movement, one or two other muscles/movements could be tested. These can be included in the 'empty' row. The common 
finger extensors are appropriate for this. The subject is asked to extend the fingers at the mcp joints keeping the fingers flexed at the IP joints. The examiner can test simultaneously all four fingers for resistance with four fingers of his hand.

It should be noted that the intrinsic thumb muscles contribute to thumb IP extension. In isolated radial nerve damage thumb IP extension may therefore still be possible and strong. This if often mistaken as an action of the extensor pollicis longus!

\section{Common peroneal nerve}

The common peroneal or lateral popliteal nerve 'branches' at the site where it winds around the head of the fibula. Depending on the exact site of branching and extent of damage one or both of the branches may be impaired. The deep branch innervates the dorsiflexors, tibialis anterior, and the two toe extensors; the superficial branch, the evertors. It is common to talk about a total footdrop when both branches are not functioning and an incomplete footdrop when only one of the two is affected, most commonly the deep branch. An irregular 'footdrop' (better irregular pattern of paralysis) would be one in which only one or two muscles are functioning or are weak, e.g. isolated weakness of toe extension or normal strength for great toe extension only.

For screening purposes, we recommend that dorsiflexion of the foot is tested. Only when weakness or paralysis of dorsiflexion is evident may further detailed testing be indicated.

\section{Posterior tibial nerve}

The posterior tibial nerve is commonly affected behind and above the medial malleolus, in or above the tarsal tunnel. For many people it is difficult to isolate a specific movement by which the strength of the intrinsic muscles can be graded. In Karigiri the technicians for many years have tested the 'toe-grip'. The subject was asked to try to keep a piece of paper between the great toe and the second toe. The examiner then tried to pull the piece of paper from between the toes. This test has now been discarded because the technicians found this test not to be very practical and stated that many times in unaffected feet with normal sensation and in control subjects the test was (false) 'positive'. Likewise, toe-fanning, the spreading of toes, is a qualitative test which is likely to be positive in many non-affected subjects!

'High' posterior tibial (medial popliteal) nerve damage very rarely occurs, some even deny its occurrence. ${ }^{15}$ High posterior tibial motor function impairment would result in weakness or paralysis of the muscles of the calf. In Karigiri, we have included a test for high posterior motor function impairment for research purposes. What is the incidence of 'high' posterior nerve impairment and is it associated with motor function impairment of the lateral popliteal nerve?

\section{Reliability}

Only a few reliability studies have been conducted on manual muscle strength testing with leprosy patients as subjects. ${ }^{16-19}$ Overall, the studies report acceptable reliability and support 
the use of manual muscle strength testing. A notable exception is the study by Lewis, which was methodologically and conceptually flawed.

In the most recent reported study, however, it has been shown that reliability coefficients to a great extent are influenced by the high proportion of completely normal or completely paralysed muscles. ${ }^{17}$ In this study intertester reliability was assessed from data obtained from 72 leprosy affected persons using an extended 9-point MRC scale. When omitting the normal scores and the completely paralysed muscle scores from the analysis, the direct agreement percentage dropped considerably. On the basis of the results of this study, the hospital in which the study was conducted decided not to 'value' the 'in-between grades' as they are sometimes reported by the physiotherapist or technician.

\section{Basic form and lay-out}

Figure 1 shows the muscle charting form with the tests recommended to screen subjects for possible motor function impairment and to monitor subjects with confirmed motor function impairment. First, the facial nerve is listed, followed by the nerves in the upper extremity and finally, the lower extremity. For the upper extremity the nerves are listed in the order of most commonly to least often affected. For each nerve, when applicable, first the intrinsic tests are listed and then the test for extrinsic muscles.

The nerves and muscles/movements are listed in the central column. On the left side of the form, the tests for the right side of the body are graded and on the right side, those for the left side. Normally, the subject is facing the examiner and so the side that is examined corresponds with the same side of the form. The results of the first assessment could be recorded starting from the centre of the form and then working towards the right and left side for subsequent assessments. Alternatively, in some programs the grades are recorded from left to right; the results of the first assessment for the right side are recorded in the first column.

The proposed form allows for seven muscle assessments. In Green Pastures Hospital, this matches the number of sensory assessments that can be recorded on the reverse side of the form.

There is a provision for comments that should be used to record the presence of pain on testing that will influence the muscle score. This should also be used to make note of irregular pattern of paralysis, duration of palsy, lack of co-operation of patient in testing etc.

The tests for screening purposes area shaded on the muscle charting form. If practical, and when expertise is available, it is recommended that 'detailed' testing is done for those nerves for which the screening test showed weakness/paralysis.

The lay-out of the form allows for easy recognition and visualization of the problem nerve(s). The row in line with the nerve headings 'separates' the nerves. This row could for example be used to indicate start and finish of prednisolone treatment. Visualization of problem nerves and changes can be further enhanced by using a red pen to record the grades for tests that score less than grade 5 on the first assessment. For subsequent assessment 'red' should only be used when there is new or increased motor function impairment.

\section{Discussion}

A notable feature of the form is the avoidance of Latin names for muscles. This will make it easier to teach muscle testing to the 'paramedical' leprosy workers. The point has been made 
that most muscles cannot be tested in isolation. Also in other patient populations in which muscle scores are an outcome measure, the researchers test and grade movements rather then individual muscles. ${ }^{20-22}$ This author proposed already in 1981 a simplification of muscle charting for leprosy patients but in many projects detailed testing is still practised. ${ }^{4}$ Goodwin already recognized in the first paper on muscle testing in leprosy that some muscles may be easy to test and that others are difficult to grade. ${ }^{1}$

The intrinsic muscles do contribute to grip and pinch strength. Some studies are underway to see if dynamometry is more sensitive in detecting and monitoring changes in the motor function of the ulnar nerve. This would be especially useful in the 3-5 MRC range. Dynamometry for the evaluation of motor function in leprosy patients was already suggested in 1963 by Harris, who gives credit for this to Dr P. Brand. ${ }^{23}$ In a case report dynamometry values and MRC scores were obtained and compared in a case of bilateral low ulnar neuropraxia. ${ }^{13}$ The empty rows on the form could be used to record grip and/or pinch strength values. Some studies look into the possible value of testing for endurance, the number of contractions with some predetermined amount of resistance, to evaluate the motor function of especially the ulnar nerve. This could be valuable to quantitate muscle strength in patients with a grade of more than 3 . These studies seek to develop a test which may be more sensitive to detect changes in motor function of the ulnar nerve.

Contraction of the interossei in the so-called lumbrical test can be easily demonstrated on the index and little fingers. When giving resistance towards flexion in the intrinsic position of these fingers, palpation of the first dorsal interosseus and abductor digiti minimi (which could be considered a dorsal interosseus) reveals a strong contraction of these muscles. These muscles are not only abductors of those fingers but they are also the primary flexors. In leprous neuropathy it is not really necessary to test all the dorsal and palmar interossei in abduction and adduction of the fingers. Besides the fact that they are already graded in the intrinsic test, abduction and adduction would be difficult to grade using the MRC grading, except, of course, in abduction of the index finger.

High median nerve function impairment (cubital fossa) does occur in leprosy patients, but is relatively rare. If present, it will be associated with weakness or paralysis of the intrinsic muscles. Weakness or paralysis of only the extrinsic muscles may occur when there is entrapment of the anterior interosseus nerve as it passes through the pronator teres but this is distal to the site where the nerve may become affected in leprosy. Nothing is known about the prevalence of high median involvement, but when present, it is usually associated with radial nerve motor function impairment. Isolated radial nerve damage does occur but a crutch palsy should be excluded. More commonly, when there is paralysis of the radial innervated muscles, there will be motor function impairment of the ulnar and median nerve as well (triple palsy).

It is advisable to test the extrinsic muscles/movements when the screening test for the nerve reveals weakness or paralysis. When the extrinsic muscle tests are normal they can then be excluded from follow up assessments. It is unlikely to find weakness or even paralysis of extrinsic muscles innervated by the ulnar or median nerve in the presence of normal intrinsic muscle strength. For the ulnar and median nerves the extrinsic muscle strength tests are in italics.

For research purposes it may be interesting to include grading of the strength of extensor hallucis $(\mathrm{EH})$ muscle to establish the prevalence of isolated $\mathrm{EH}$ weakness/paralysis in the presence of normal strength for dorsiflexion. It has been noticed that there may be isolated weakness and even paralysis of $\mathrm{EH} .^{17}$ 
It would be good to include in some centres in the muscle charting form a test for the motor function of the posterior tibial nerve ('high' - not shown in the muscle chart) in addition to a test for the motor function of high median nerve. This is for research purposes only, in order to be able to say something about the prevalence of high posterior tibial and high median nerve motor function impairment and its possible association with lateral popliteal and radial nerve motor impairment, respectively.

Sometimes paralysis of the $\mathrm{m}$. frontalis is noticed. This is the muscle responsible for wrinkling the skin above the eye, the forehead. The muscle is innervated by a branch of the facial nerve. The nerve that is sometimes palpable across the forehead is a branch of the ophthalmic branch of the trigeminal nerve (supra-orbital nerve). Grading of the $\mathrm{m}$. frontalis on the MRC scale is not practical. Would a count of wrinkles be useful?

Manual muscle testing is often considered to be 'subjective', implying unreliability. Whereas there is an element of subjectivity in the muscle testing, several studies have shown that muscle strength can be reliably graded. Pain, the ultimate subjective phenomenon, can be very reliably assessed. The nature of the phenomena to be tested and the reliability of a test are often confused. Objective phenomena may be measured unreliability; subjective phenomena when measured with adequate instruments can show high inter- or intratester reliability. ${ }^{24}$

Further studies on the reliability of manual muscle strength testing in leprosy are encouraged, both field and hospital based. A cautionary note: it is not correct to assume that when acceptable reliability has been reported in one or more studies, reliability will also be good in your own setting. Only a replicate study will confirm this.

\section{Acknowledgement}

I would like to thank Dr Wim van Brakel for helpful comments on a previous draft of this manuscript.

\section{References}

${ }^{1}$ Goodwin CS. The use of the voluntary muscle test in leprosy neuritis. Lepr Rev, 1968; 39: 209-216.

2 Brunnstrom S. Muscle group testing. Physiother Rev, 1941; 21: 3-21.

3 Basmajian JV, DeLuca CJ. Muscles alive: their function revealed by electromyography, 5th edn. Williams \& Wilkins, Baltimore, 1985.

4 Brandsma JW. Basic nerve function assessment in leprosy patients. Lepr Rev, 1981; 52: 161-170.

5 The guarantors of brain: aids to the examination of the peripheral nervous system. Balliere Tindall, London, Philadelphia, 1986. (Previously; Medical Research Council, Aids to the investigation of peripheral nerve injuries. HMSO, London, 1943)

6 Bourrel P. Two objective 'archivable' tests for voluntary muscle testing in ulnar and median nerve paralysis. Ind $J$ Lepr, 1997; 69: 13-23.

7 Fritschi EP. Field detection of early neuritis in leprosy. Lepr Rev, 1987; 58: 173-177.

${ }^{8}$ Pearson JMH. The evaluation of nerve damage in leprosy. Lepr Rev, 1982; 53: 119-130.

9 Brandsma JW. Sensible manual muscle strength testing to evaluate and monitor motor function impairment of the ulnar and median nerves. $J$ Hand Ther, submitted.

${ }^{10}$ Brandsma JW, Birke JA. The Martin-Gruber innervated hand. J Hand Surg, 1986: 11A: 536-539.

11 Leibovics SJ, Hastings H. Martin-Gruber revisited. J Hand Surg, 1992; 17A: 47-53.

12 Uchida Y, Sugioka Y. Electrodiagnosis of Martin-Gruber connection and its clinical importance in peripheral nerve injury. J Hand Surg, 1992; 17A: 54-59.

13 Brandsma JW. Manual muscle strength testing and dynamometry for bilateral ulnar neuropathy in a surgeon. $J$ Hand Ther, 1995; 8: 191-194. 
14 Maimaris C, Zadeh HG. Ulnar nerve compression in the cyclists' hand: two case reports and review of the literature. Br J Sports Med, 1990; 24: 245-246.

15 Srinivasan H. Disability, deformity and rehabilitation. In: Hastings RC (ed) Leprosy, 2nd edn. Churchill Livingstone, London, Edinburgh, New York, 1994, p. 416.

16 Brandsma JW, Schreuders TAR, Birke JA et al. Manual muscle strength testing: intra- and interobserver reliability of the intrinsic muscles of the hand. J Hand Ther, 1995; 8: 185-190.

17 Brandsma JW, Brakel WH van, Andersen AM et al. Intertester reliability of manual muscle strength testing in leprosy patients. Lepr Rev, 1998; 69: 257-266.

18 Lewis S. Reproducibility of sensory testing and voluntary muscle testing in evaluating the treatment of acute neuritis in leprosy patients. Lepr Rev, 1983; 54: 23-30.

19 Lienhart C, Currie H, Wheeler JG. Inter-observer variability in the assessment of nerve function in leprosy patients in Ethiopia. Int J Lepr, 1995; 63: 62-76.

20 Andres PL, Skerry LM, Thornell B et al. A comparison of three measures of disease progression in ALS. J Neural Sci, 1996; 139: 64-70.

21 Florence JM, Pandya S, King WM et al. Intrarater reliability of manual muscle test (Medical Research Council Scale) grades in Duchenne's muscular dystrophy. Phys Ther, 1992; 72: 115-126.

22 Kleyweg RP, van der Meche FGA, Schmitz PIM. Interobserver agreement in the assessment of muscle strength and functional abilities in Guillain-Barre syndrome. Muscle Nerve, 1991; 14: 1103-1109.

${ }^{23}$ Harris JR. Criteria for assessing the results of acute ulnar neuritis in leprosy. Lepr Ind, 1964; 35: 107-109.

24 Rothstein JM, Echternach JL. An introductory guide to measurement issues. American Physical Therapy Association, Alexandria, 1993. 